\title{
Effect of Curvature of Tip and Convexity of Electrode on Localization of Particles
}

\author{
Sudarshan Ghonge1, D. Nagendra Prasad', Swarnim Narayan'1, Hains Francis', \\ Astha Sethi' ${ }^{2}$, Subimal Deb ${ }^{3}$, Souri Banerjee ${ }^{1}$ \\ ${ }^{1}$ Department of Physics, Birla Institute Technology and Science-Pilani, Hyderabad Campus, \\ Hyderabad, India \\ ${ }^{2}$ Department of Physics and Frederick Seitz Materials Research Laboratory, University of Illinois, \\ Urbana-Champaign, IL, USA \\ ${ }^{3}$ Department of Physics, GIT, GITAM University, Visakhapatnam, India \\ Email: souri@hyderabad.bits-pilani.ac.in
}

Received 9 October 2015; accepted 30 November 2015; published 3 December 2015

Copyright @ 2015 by authors and Scientific Research Publishing Inc.

This work is licensed under the Creative Commons Attribution International License (CC BY). http://creativecommons.org/licenses/by/4.0/

(c) (i) Open Access

\begin{abstract}
We investigate the effect of curvature of the tip and the convexity of an electrode on the localization of suspended particles under the combined effect of dielectrophoresis and AC electroosmosis through simulations using COMSOL Multiphysics. A systematic analysis of the parameters defining the convexity of the electrode-the radius of the tip and the apex angle shows that suspended particles can be trapped closely to the electrode edges for comparatively larger tip radii and apex angles. This in turn should favour the trapping of polarizable molecules between the electrodes only if the fluid velocities at the vortices are not very strong.
\end{abstract}

\section{Keywords}

Finite Element Method, Transport Dynamics, AC Electroosmosis, Dielectrophoresis, Vortex

\section{Introduction}

The manipulation of polarizable biomolecules like DNA by electrical forces in microdevices has become a topic of increasing interest as it finds wide-spread applications in lab-on-a-chip technology or in DNA diagnostics [1]. In a suitable condition, a DNA molecule in an electrolytic solution will develop a strong electric dipole moment [2]. This induced dipole allows trapping and manipulation of the molecule when placed within micro-electrodes owing to a process known as dielectrophoresis (DEP) [3] [4]. In this context it is worth mentioning that the dielectrophoretic motion of DNA molecules requires alternate current (AC) electric field to suppress the electro-

How to cite this paper: Ghonge, S., Prasad, D.N., Narayan, S., Francis, H., Sethi, A., Deb, S. and Banerjee, S. (2015) Effect of Curvature of Tip and Convexity of Electrode on Localization of Particles. Open Journal of Fluid Dynamics, 5, 295-301.

http://dx.doi.org/10.4236/ojfd.2015.54030 
phoric effect of the molecules' net charge [5]. However, the motion of the DNA molecules and hence their trapping/manipulation possibilities within a set of microelectrodes will also be countered by the flow of the electrolyte known as AC electro-osmosis (ACEO) [6]. Thus balancing DEP and ACEO is found to be a promising technique to study non-contact manipulation/trapping of DNA molecules suspended in an electrolyte solution and placed within electrically fed microelectrodes [7]. Washizu et al. [8] used a set of interdigitated sinusoidally corrugated aluminum microelectrodes to experimentally observe site-specific immobilization of double-stranded DNA molecules under AC electric field and concluded that the orientation of DNA molecules depended strongly on the electric field strength. Similar interdigitated rectangular metal microelectrodes of different dimensions and gap lengths were later investigated, both experimentally and numerically, by several researchers [9] in order to understand movement of DNA molecules within microelectrodes under electrokinetic effects. From these studies it appears that one might further exploit state-of-art lithographic technique to fabricate smaller electrodes with sharper bends which would intensify the non-uniformity of electric field and significantly influence DNA manipulation. To this end, use of triangular shaped electrodes has recently been very popular [10] [11]. Porath et $a l$. [10] reported the first experimental evidence of trapping of a single, double stranded DNA molecule between the two vertices of a set of triangular electrodes where a few nanometers were apart. It clearly revealed that electric field strength which was extremely large at the vertices of the triangular electrode, coupled with polarization properties, played an important role in guiding the flow of DNA molecules in and around the tips. However, the most modern lithographic technique has its limitation to reproduce the bends or sharpness of the electrodes [11]. Thus it is interesting to study how small variations in electrode configurations would affect the nature of immobilization of the suspended DNA molecules confined between them.

In this report, we present a systematic analysis of the effect of curvature of the electrode tips on the localization of insoluble particles. We have also tried to assess how the convexity of the electrodes could influence the localization process.

\section{Theory}

The system under consideration consisting of two triangular electrodes is schematically represented in Figure 1. The vertices of the triangular electrodes are rounded as would be in the case of an actual fabrication. The rounded vertex results in a lower gradient of electric field around the tip in contrast to sharp vertices. We shall call the radius $r$ of the rounded electrode corner (represented by a dashed circle) the fillet radius. The vertex angle $\theta$ (acute angle between the electrode edges) defines the convexity of the electrodes. An increase in $r$ keeping $\theta$ constant would result in electrodes with blunter tips with the same convexity. This is to match a practical scenario where lithographic tools are used to fabricate microlectrodes [11]. Such a pair of electrodes placed at the base of a cubical region filled with an electrolyte, is studied numerically for the possibility of localization of suspended particles.

The properties of the electrolyte used for this configuration correspond to that of $\mathrm{KCl}$ at infinite dilution with electrical conductivity of $0.0021 \mathrm{~S} / \mathrm{m}$, relative permittivity of 78.49 , density of $1000 \mathrm{~kg} / \mathrm{m}^{3}$ and a dynamic viscosity (at $293 \mathrm{~K}$ ) of $0.001 \mathrm{~Pa}-\mathrm{s}$ [12].

We consider the variation of the apex angle with a fixed separation $(d)$ between the tips of the electrode. The

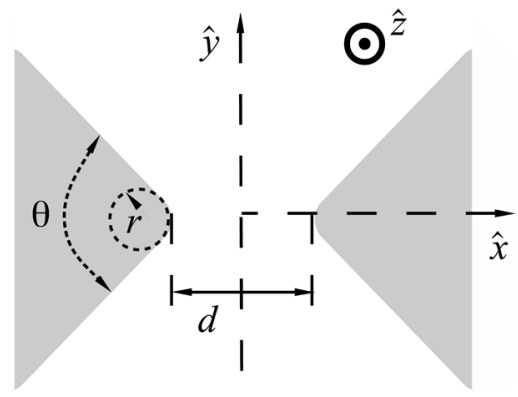

Figure 1. Schematic representation of the top view of the electrode setup. The dashed lines along $\hat{x}$ and $\hat{y}$ represent the planes of mirror symmetry transverse to the plane of the electrodes. 
$y-z$ plane, a plane of mirror symmetry between the electrodes, reduces the domain of the problem by half owing to the symmetry of the configuration and opposite polarities of the electrodes. Clearly an obtuse apex angle is a concave configuration of the electrode edges facing each other. Convex electrodes produce the strongest electric fields near their apices owing to the proximity of the tips. This produces a region of strong electric field at the center of the configuration that gradually decreases away from the origin along the y-axis on either side. A concave electrode, on the other hand, has a gradually decreasing spacing between the edges (measured along the $\mathrm{x}$-axis) which produces a field of least strength at the origin, gradually increasing along the positive and negative $y$-axis. Changing the apex angle from acute to obtuse is a cross-over from the first regime of the electric field decreasing away from the origin to the one where the field density change is reversed. We cover both the regimes by changing the apex angle.

The special case of plane parallel electrodes $(\theta=\pi)$ exhibits two symmetries. The first is a translational symmetry along the edge of the electrodes. The second is a mirror symmetry about the $y$-z plane through the origin. The mirror symmetry warrants the use of the method of images under an applied electric potential and reduces the problem space by half. The translational symmetry reduces the configuration to two dimensions confined to the plane of translational symmetry. This configuration was studied in detail by Green et al. [13].

The arrangement of triangular electrodes has two planes of mirror symmetry in the arrangement: the first being the $x-z$ plane passing through the apex of both the electrodes; the second being the y-z plane through the origin. This space, however, must be studied as a three-dimensional system since the convexity of the electrodes breaks the translational symmetry that existed for $\theta=\pi$. The three parameters for our study could be $r, \theta$ and $d$, where one could systematically vary a parameter holding the other two fixed. Here we present the simulation studies with varying $\theta$ keeping $r$ constant and vice-versa for a fixed value of $d$. The simulations were performed using COMSOL Multiphysics version 4.3a [14]. The method of images is used for the analysis of the system owing to the antisymmetry of the applied potential across the $y$-z plane which will be at a potential of $0 \mathrm{~V}$. To study the effect of AC electroosmosis, the double layer was modelled as distributed impedance over the electrode surface. The potential applied on the surface of the electrodes is

$$
V(t)=V_{e p} \sin \omega t
$$

We use $V_{e p}=0.25 \mathrm{~V}$ with the applied potential having an angular frequency $\omega=5 \mathrm{kHz}$. The distributed impedance model defines the spatial variation of the potential $\left(\phi_{e p}\right)$ at the edges of the double layer formed

$$
\sigma \nabla \phi_{e p}=\frac{\phi_{e p}-V_{e p}}{Z_{D L}} ; Z_{D L}=\frac{1}{i \omega C_{D L}}
$$

where $\sigma$ is the electrical conductivity, $C_{D L}$ is the capacitance of the double layer. The components of the flow velocities are computed using Navier-Stokes equations to identify regions capable of localizing or trapping particles around the electrodes. The time averaged DEP force for a spherical particle of radius $R$ is given by the expression [15]

$$
F_{\text {dep }}=2 \pi \epsilon_{m} R^{3} \mathfrak{R}\{[K(\omega)]\} \nabla\left|E_{\text {rms }}\right|^{2}
$$

where the Clausius-Mossotti factor

$$
K(\omega)=\frac{\tilde{\epsilon}_{p}-\tilde{\epsilon}_{m}}{\tilde{\epsilon}_{p}+2 \tilde{\epsilon}_{m}} ; \tilde{\epsilon}_{p, m}=\epsilon_{p, m}-\frac{i \sigma_{p, m}}{\omega}
$$

Here $\epsilon$ and $\sigma$ are the complex permittivities and conductivities respectively with subscripts $p$ and $m$ referring to the particle and medium respectively. We choose $\epsilon_{p}=4 \epsilon_{0}, \sigma_{p}=2.4 \mathrm{~S} \cdot \mathrm{cm}^{-1}$ at $25^{\circ} \mathrm{C}, K(5 \mathrm{kHz})=$ $0.9999-1.3345 \times 10^{-7} i$.

The radius of the Lambda DNA is known to be in the range 2 to $4 \mu \mathrm{m}$. A similar study by Bown and Meinhart [16] on the DNA of Lambda phage bacteria was reported in a frequency range of $100 \mathrm{~Hz}$ to $1.6 \mathrm{MHz}$ with a rotationally symmetric electrode configuration. The expression for the DEP force on a particle, also given by an alternate expression [3], provides a measure of the effective polarizability of the particle and the magnitude of the electric field, which has been used in earlier simulations [17] and the formula as given by Equation 3 is found suitable to calculate DEP forces for spherical particles [15]. Washizu and Kurosawa [2] have shown that 
the DNA would stretch under an electric field. Stretching the DNA can result in a change of the effective polarizability of the molecule resulting in a shift of the trapping points. Regtmeier et al. [18] have reported that the polarizability of the DNA in linear and supercoiled forms is of similar magnitude within error margins. We therefore use the same effective polarizability for free and trapped particles assuming that the stretched DNA (under the applied field) will not affect the trapping points.

\section{Results and Discussion}

We use a particle size of $R=4 \mu \mathrm{m}$ to study the feasibility of polarized and stretched DNA molecules forming a bridge across electrodes. The forces on the stretched DNA molecule are the DEP force (calculated from the computed electric fields) and the drag force (calculated from Stoke's law). Figure 2 shows the variation of the $x$-component of the force on a stretched DNA molecule along $x$-axis for an electrode system having typical parameters $d=25 \mu \mathrm{m}, r=0.5 \mu \mathrm{m}$, and $\theta=102^{\circ}$. It is important to point out that at any point of time the electrodes under the application of an alternating voltage have opposite polarities. The symmetry of the electrode configuration and the simultaneous antisymmetry of the electric potential exist for all ranges of parameters $r, d$ and $\theta$. The range of parameters examined show the same nature of the x-component of the force. Here we present only a typical plot of the force for $d=25 \mathrm{~m}, r=0.5 \mathrm{~m}, \theta=102^{\circ}$. Therefore Figure 2 is representative of any electrode configuration defined by the parameters $d, r$ and $\theta$. It is worth noting from the plot that the force has a positive and a negative value on either side of a point where the force vanishes. Such a force can represent only a vortex. The suspended particles in the electrolyte upon entering the vortex get trapped in it due to the torque set up by the vortex. The results show that the vortex is located near the edge of the electrode and therefore the insoluble particles in the vortex will have a higher probability of bridging the gap between the electrodes. This also requires that the torque on the trapped particles should not be too great to prohibit it from exiting the vortex.

\subsection{Variation of Apex Angle}

A variation of $\theta$ for a fixed $r$ and electrode separation leads to increasing electric field density with decreasing $\theta$. Quickly varying electric field densities indicate the existence of strong vortices with a short span along the $x$-axis, as envisaged from Figure 2 as well. We test it for four different angles keeping $r=0.5 \mu \mathrm{m}$ and $d=25 \mu \mathrm{m}$.

Figure 3 shows the variation of the slope (stiffness) of the $x$ component of the force as a function of $\theta$ for the convex (dashed curve) and the concave (solid curve) electrodes where the magnitude of the slope is taken as a measure of the stiffness. The plot clearly shows a systematic increase of the stiffness of the force with increasing $\theta$ for the set of concave electrodes. It should be noted that in Figure 3. We have plotted the slope of the $\mathrm{x}$-component of the force for the two cases namely, convex and concave electrodes. It is worthwhile to mention that angle in the plot is the acute angle between the edges of the electrode. Hence, the angle measured for the concave electrode, if measured on the same side of the electrode edge as for the convex electrode, would be a

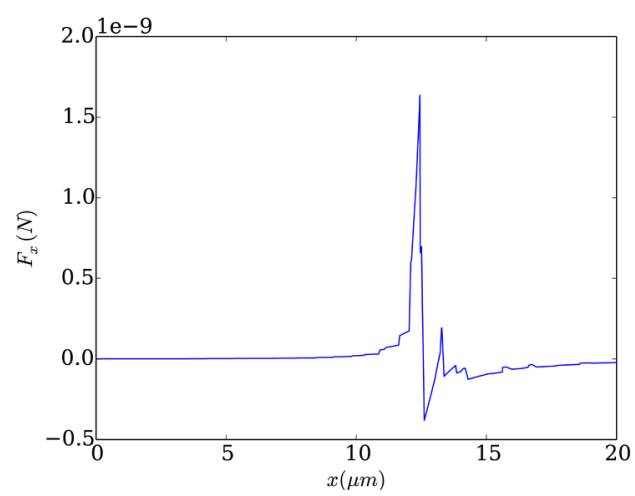

Figure 2. Variation of the $x$-component of the force on a stretched DNA molecule along the x-axis. The specific parameters used are $d=25 \mu \mathrm{m}, r=0.5 \mu \mathrm{m}, \theta=102^{\circ}$. 


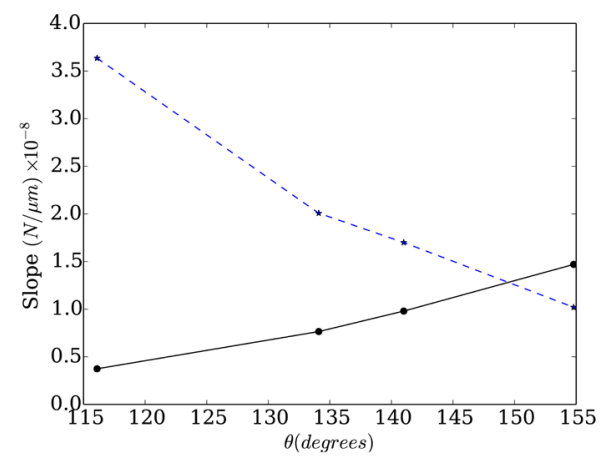

Figure 3. Variation of the slope of the $x$-component of the force as a function of the apex angle $\theta$ for the concave (solid curve) and convex (dashed curve) electrodes

reflex angle, and therefore, the two plots in Figure 3 are consistent with each other. Smaller slope (stiffness) at larger $\theta$ indicates that electrodes with less convexity is likely to generate vortex sites of weaker trapping capacity. This prompts us to further investigate the scenario with electrodes having moderate convexity but with varying apex radius as it would address a more practical issue. It is well accepted that lithographically fabricated micro/nano electrodes would yield rounded tips of different curvature due to the resolution limit of the respective lithographic tool used [10] [11].

\subsection{Variation of Apex Radius}

A variation of the fillet radius $r$ changes the electric field density around the tip of the electrodes. A sharper electrode tip produces a stronger electric field and strengthens the DEP force. Thus, the net force across the vortex is expected to change faster for sharper electrodes. We test this by plotting the variation of $x$-component of the force $F_{x}$ as a function of the fillet radius $r$ at the center of the vortex keeping electrode separation $d=25 \mu \mathrm{m}$ and apex angle $\theta=102^{\circ}$. Figure 4 shows the variation of the slope of the $x$-component of the force as a function of $r$ for the convex (dashed curve) and the concave (solid curve) electrodes. Specifically, the slope was calculated by taking a linear approximation of the curve between the two points where the force reaches its extremal values. $F_{x}$ varied at a rate of $0.48 \times 10^{-10} \mathrm{~N} / \mu \mathrm{m}$ for flat electrodes $(r \rightarrow \infty)$. The decrease of the stiffness of the force with the fillet radius occurs for all angles checked in the range of $116.1^{\circ}$ to $154.8^{\circ}$. The plot (Figure 4(a)) suggests that electrodes with smaller $r$ (i.e. having sharper tip) would produce stronger vortices within the electrolyte. Keeping in mind real life fabrication issues, the strength and the span of the vortex are of interest to us. We calculate the field strength by taking the average of the magnitude of the extremal values of $F_{x}$ and the span is calculated by finding the spacing between the locations where $F_{x}$ takes the extremal values. Figure 4(b) and Figure 4(c) represent the variation of the strength of the field and span respectively as a function of fillet radius. From the plots it is clear that the strength decreases and the span increases as the electrode tips become more and more blunt.

\section{Conclusion}

In conclusion, we have systematically analyzed two of the three parameters (the fillet radius and the apex angle) that may be tuned to have a localizing region for suspended DNA molecules in electrolytes. It is found that stiffness of the force decreases with increase of the apex angle, indicating weaker trapping sites in the electrolyte as the convexity of the electrode increases. In this context we tried to address a practical situation where electrodes of fixed and moderate convexity but having different curvature of the tips were considered. Our study reveals that the strength of the vortex gets weaker and its span becomes wider as the tip gets more and more rounded. It also provides an intuitive insight that, if polarization of DNA molecules has any role to facilitate the DNA attachment process, it is desirable to have vortex of moderate strength and span around the edge of the electrode such that the molecules are localized (rather than being strongly trapped) in the vortex facilitating polarization to complete the bridging process. Therefore, it is suggested that a set of electrodes with fixed convexity but having rounded tips may better serve the purpose of bridging electrodes by DNA molecules. It would be 


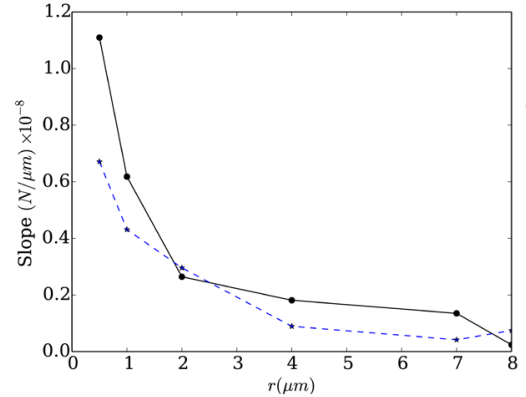

(a)

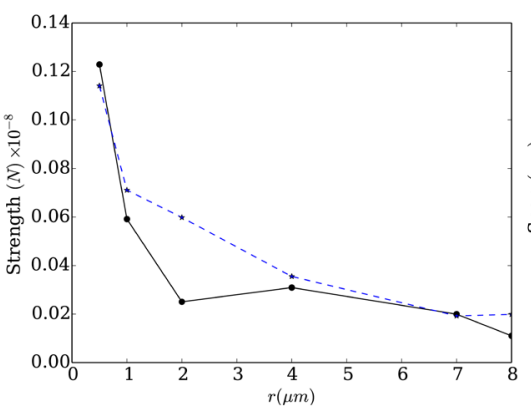

(b)

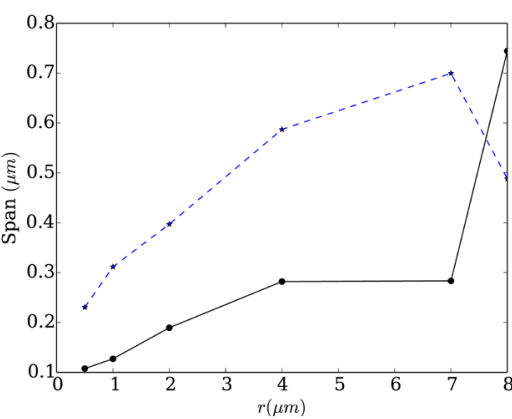

(c)

Figure 4. Variation of the (a) slope, (b) strength and (c) span of the $x$-component of the force as a function of the fillet radius $r$ for the concave (solid curve) and convex (dashed curve) electrodes.

interesting to further study the strength, distribution and the span of vortex points within the electrolyte as a function of electrode separation as well (keeping $r$ and $\theta$ constant), which is already underway.

\section{Acknowledgements}

One of the authors (SB) acknowledges the financial support of the Board of Research in Nuclear Sciences, Department of Atomic Energy, Govt. of India for financial assistance.

\section{References}

[1] Abramowitz, S. (1996) Towards Inexpensive DNA Diagnostics. Trends in Biotechnology, 14, 397-401. http://dx.doi.org/10.1016/0167-7799(96)10051-2

[2] Washizu, M. and Kurosawa, O. (1990) Electrostatic Manipulation of DNA in Microfabricated Structures. IEEE Transactions on Industry Applications, 26, 1166-1172. http://dx.doi.org/10.1109/28.62403

[3] Pohl, H.A. (1978) Dielectrophoresis: The Behavior of Neutral Matter in Nonuniform Electric Fields. Cambridge University Press, Cambridge.

[4] Asbury, C.L. and van den Engh, G. (1998) Trapping of DNA in Nonuniform Oscillating Electric Fields. Biophysical Journal, 74, 1024-1030. http://dx.doi.org/10.1016/s0006-3495(98)74027-5

[5] Bakewell, D.J. and Morgan, H. (2006) Dielectrophoresis of DNA: Time- and Frequency-Dependent Collections on Microelectrodes. IEEE Transactions on Nano Bioscience, 5, 1-8.

[6] Loucaides, N.G., Ramos, A. and Georghiou, G.E. (2008) Trapping and Manipulation of Nanoparticles by Using Jointly Dielectrophoresis and AC Electroosmosis. Journal of Physics: Conference Series, 100, Article ID: 052015. http://dx.doi.org/10.1088/1742-6596/100/5/052015

[7] Du, J.-R., Juang, Y.-J., Wu, J.-T. and Wei, H.-H. (2008) Long-Range and Superfast Trapping of DNA Molecules in an AC Electrokinetic Funnel. Biomicrofluidics, 2, Article ID: 044103. http://dx.doi.org/10.1063/1.3037326

[8] Washizu, M., Kurosawa, O., Arai, I., Suzuki, S. and Shimamoto, N. (1995) Applications of Electrostatic Stretch-andPositioning of DNA. IEEE Transactions on Industry Applications, 31, 447-456. http://dx.doi.org/10.1109/28.382102

[9] Asbury, C.L., Diercks, A.H. and van den Engh, G. (2002) Trapping of DNA by Dielectrophoresis. Electrophoresis, 23, 2658-2666. http://dx.doi.org/10.1002/1522-2683(200208)23:16<2658::aid-elps2658>3.0.c0;2-0

[10] Porath, D., Bezryadin, A., De Vries, S. and Dekker, C. (2000) Direct Measurement of Electrical Transport through DNA Molecules. Nature, 403, 635-638.

[11] Zheng, L.F., Brody, J.P. and Burke, P.J. (2004) Electronic Manipulation of DNA, Proteins, and Nanoparticles for Potential Circuit Assembly. Biosensors and Bioelectronics, 20, 606-619. http://dx.doi.org/10.1016/j.bios.2004.03.029

[12] Ramos, A., Morgan, H., Green, N.G. and Castellanos, A. (1998) AC Electrokinetics: A Review of Forces in Microelectrode Structures. Journal of Physics D: Applied Physics, 31, 2338. http://dx.doi.org/10.1088/0022-3727/31/18/021

[13] Green, N.G., Ramos, A., Gonzalez, A., Morgan, H. and Castellanos, A. (2002) Fluid Flow Induced by Nonuniform AC Electric Fields in Electrolytes on Microelectrodes. III. Observation of Streamlines and Numerical Simulation. Physical Review E, 66, Article ID: 026305. http://dx.doi.org/10.1103/PhysRevE.66.026305

[14] Comsol 4.3a. http://www.comsol.com 
[15] Green, N.G. and Morgan, H. (1997) Dielectrophoretic Investigations of Sub-Micrometre Latex Spheres. Journal of Physics D: Applied Physics, 30, 2626. http://dx.doi.org/10.1088/0022-3727/30/18/018

[16] Bown, M.R. and Meinhart, C.D. (2006) AC Electroosmotic Flow in a DNA Concentrator. Microfluidics and Nanofluidics, 2, 513-523. http://dx.doi.org/10.1007/s10404-006-0097-4

[17] Tuukkanen, S., Kuzyk, A., Toppari, J.J., Häkkinen, H., Hytönen, V.P., Niskanen, E., Rinkiö, M. and Törmä, P. (2007) Trapping of $27 \mathrm{bp}$ - 8 kbp DNA and Immobilization of Thiol-Modified DNA Using Dielectrophoresis. Nanotechnology, 18, Article ID: 295204. http://dx.doi.org/10.1088/0957-4484/18/29/295204

[18] Regtmeier, J., Eichhorn, R., Bogunovic, L., Ros, A. and Anselmetti, D. (2010) Dielectrophoretic Trapping and Polarizability of DNA: The Role of Spatial Conformation. Analytical Chemistry, 82, 7141-7149.

http://dx.doi.org/10.1021/ac1005475 